\title{
Potential Usefulness of Streptococcus pneumoniae Extracellular Membrane Vesicles as Antibacterial Vaccines
}

\author{
Chi-Won Choi, ${ }^{1,2}$ Edmond Changkyun Park, ${ }^{1,3,4}$ Sung Ho Yun, ${ }^{1}$ Sang-Yeop Lee, ${ }^{1,4}$ \\ Seung Il Kim, ${ }^{1,3,4}$ and Gun-Hwa Kim ${ }^{1,2,5}$ \\ ${ }^{1}$ Division of Bioconvergence Analysis, Korea Basic Science Institute, Daejeon, Republic of Korea \\ ${ }^{2}$ Tunneling Nanotube Research Center, Division of Life Science, Korea University, Seoul 02841, Republic of Korea \\ ${ }^{3}$ Department of Bio-Analytical Science, University of Science and Technology, Daejeon, Republic of Korea \\ ${ }^{4}$ Center for Convergent Research of Emerging Virus Infection, Korea Research Institute of Chemical Technology, \\ Daejeon, Republic of Korea \\ ${ }^{5}$ Department of Functional Genomics, University of Science and Technology, Daejeon, Republic of Korea
}

Correspondence should be addressed to Seung Il Kim; ksi@kbsi.re.kr and Gun-Hwa Kim; genekgh@kbsi.re.kr

Received 30 September 2016; Revised 24 November 2016; Accepted 21 December 2016; Published 22 January 2017

Academic Editor: Giuseppe A. Sautto

Copyright (C) 2017 Chi-Won Choi et al. This is an open access article distributed under the Creative Commons Attribution License, which permits unrestricted use, distribution, and reproduction in any medium, provided the original work is properly cited.

\begin{abstract}
The secretion of extracellular membrane vesicles (EMVs) is a common phenomenon that occurs in archaea, bacteria, and mammalian cells. The EMVs of bacteria play important roles in their virulence, biogenesis mechanisms, and host cell interactions. Bacterial EMVs have recently become the focus of attention because of their potential as highly effective vaccines that cause few side effects. Here, we isolated the EMVs of Streptococcus pneumoniae and examined their potential as new vaccine candidates. Although the S. pneumoniae bacteria were highly pathogenic in a mouse model, the EMVs purified from these bacteria showed low pathological activity both in cell culture and in mice. When mice were injected intraperitoneally with $S$. pneumoniae EMVs and then challenged, they were protected from both the homologous strain and another pathogenic serotype of S. pneumoniae. We also identified a number of proteins that may have immunogenic activity and may be responsible for the immune responses by the hosts. These results suggest that $S$. pneumoniae EMVs or their individual immunogenic antigens may be useful as new vaccine agents.
\end{abstract}

\section{Introduction}

Streptococcus pneumonia is an alpha-hemolytic Gram-positive encapsulated aerobic diplococcus bacterium that is the main causative pathogen of community-acquired respiratory tract infections. S. pneumoniae is generally considered to be a human pathogen because it causes a number of human diseases, including otitis, sinusitis, bacterial meningitis, sepsis, and pneumonia [1]. The people who are most affected by this organism are children and individuals with immature or compromised immune systems, such as patients with diabetes or acquired immunodeficiency syndrome $[2,3]$. Moreover, S. pneumoniae has been isolated from various animals, including guinea pigs, cats, horses, dogs, and gorillas. These animals all exhibit $S$. pneumoniae-related clinical symptoms [4]. These S. pneumonia-infected animal hosts thus may serve as an extrahuman reservoir from which the pathogen can be transmitted to humans. Therefore, to prevent $S$. pneumoniae infections, it is necessary to vaccinate both humans and animals such as pets. Pneumococcal conjugate vaccines are widely used because they are highly effective in preventing pneumococcal invasive disease. A recent review reported that failure of pneumococcal conjugate vaccines is rare, but irrespective of vaccine or schedule [5]. This may lead us to evaluate EMVs of S. pneumoniae for vaccine candidates.

Bacterial extracellular membrane vesicles (EMVs) are spherical vesicles that are secreted by a variety of bacteria. These vesicles measure $20-250 \mathrm{~nm}$ in diameter and contain various biologically active proteins that are required for bacterial nutrient acquisition, biofilm formation, and pathogenesis [6]. Since bacterial EMVs are nonviable and yet induce a host immune response, they have great potential 
as acellular antibacterial vaccines. In particular, the EMV surface proteins can act as antigens that induce adaptive immune responses in the host.

Here, we isolated nonpathogenic (noncapsular) S. pneumoniae EMVs and examined their ability to serve as nextgeneration vaccines that protect against infections with pathogenic or nonpathogenic bacteria. We also identified the antigenic protein components of the EMVs.

\section{Materials and Methods}

2.1. Bacterial Strains and Growth Conditions. Streptococcus pneumoniae BAA-255 was purchased from the American Type Culture Collection (ATCC, http://www.atcc.org, Manassas, VA, USA). Streptococcus pneumoniae KCCM-41569 was obtained from the Korean Culture Center of Microorganisms (KCCM, http://www.kccm.or.kr). The bacteria were grown to an $\mathrm{OD}_{600}$ of approximately 1.0 in a $5 \% \mathrm{CO}_{2}$ atmosphere at $37^{\circ} \mathrm{C}$ in Todd-Hewitt broth supplemented with $0.5 \%$ yeast extract and bacteria cells were counted by using Quantum Tx microbial cell counter (Logos Biosystems, Korea).

\subsection{Purification of EMVs. EMVs of S. pneumoniae BAA-255} were purified from bacterial culture supernatants by using the method of Choi et al. [7], with some modifications. Briefly, the bacterial cells were removed from the bacterial culture by centrifugation at $16,000 \times \mathrm{g}$ for $20 \mathrm{~min}$. The supernatants were then filtered through a $0.2 \mu \mathrm{m}$ hollow fiber membrane (GE Healthcare, Little Chalfont, Buckinghamshire, UK) to further remove residual cells and debris. The EMVs were concentrated and ultrafiltrated by using a QuixStand Benchtop System fitted with a $500 \mathrm{kDa}$ hollow fiber membrane (GE Healthcare). The resulting EMVs were precipitated by ultracentrifugation at $150,000 \times \mathrm{g}$ for $3 \mathrm{~h}$ at $4^{\circ} \mathrm{C}$ and the EMV-containing pellets were suspended in $1.0-2.0 \mathrm{~mL}$ of phosphate-buffered saline (PBS). Finally, the EMV solution was layered over a sucrose gradient $(2.5,1.6$, and $0.6 \mathrm{M}$ sucrose) to remove contaminating proteins. Each fraction was centrifuged at $200,000 \times \mathrm{g}$ for $20 \mathrm{~h}$ at $4^{\circ} \mathrm{C}$ and the sucrose was removed by ultracentrifugation at $150,000 \times \mathrm{g}$ for $3 \mathrm{~h}$ at $4^{\circ} \mathrm{C}$. The protein concentration was determined by using a bicinchoninic acid assay (Thermo Scientific, Waltham, MA, USA). The purified EMVs were stored at $-80^{\circ} \mathrm{C}$ until required.

2.3. Transmission Electron Microscope (TEM) Observation of EMVs. For TEM analysis, $5 \mu \mathrm{L}$ of the $5 \mathrm{mg} / \mathrm{mL}$ purified EMV sample was loaded onto a freshly glow-discharged holey carbon EM grid (Quantifoil R 2/2, Quantifoil Micro Tools GmbH, Germany). Semiautomated sample verification was performed by using a Vitrobot Mark IV (FEI Company, Eindhoven, Netherlands) at $4^{\circ} \mathrm{C}$ and $90-100 \%$ relative humidity. The vitrified sample was imaged under low dose conditions by using a Tecnai $G^{2}$ Spirit TEM (FEI Company, Eindhoven, Netherlands) that was operated at $120 \mathrm{kV}$ acceleration voltage. Images were recorded by using an UltraScan 4000 charge-coupled device camera (Gatan Inc.,
Pleasanton, CA, USA) at a nominal magnification of $\times 26,000$ and $-1-2 \mu \mathrm{m}$ underfocus.

2.4. Viability and Apoptosis of Human Lung Cancer Cells after Treatment with Bacteria and EMVs. Briefly, the human lung epithelial adenocarcinoma line A549 was cultured in 96-well plates $\left(1 \times 10^{5}\right.$ cells/well $)$ with $S$. pneumoniae bacteria or EMVs for $24 \mathrm{~h}$, after which cell viability and apoptosis were measured as described previously [7]. The culture medium was RPMI 1640 (Gibco, Waltham, MA, USA) supplemented with heat-inactivated $10 \%$ FBS and antibiotics (Gibco). The ranges of multiplicity of infection (MOI) of S. pneumoniae that were used to infect the A549 cells were 0.001, 0.1, 10, and 1,000 for S. pneumoniae BAA-255 and 0.001, 0.01, 0.1, and 1 for S. pneumoniae KCCM-41569. The concentrations of S. pneumoniae BAA-255 EMVs were 50, 100, and $200 \mu \mathrm{g}$ protein in $100 \mu \mathrm{L}$ of culture medium. To measure cell viability, the cells were stained with acridine orange and DAPI (ChemoMetec, Allerød, Denmark). To measure apoptosis, the cells were stained with FITC-conjugated Annexin V, propidium iodide, and Hoechst (ChemoMetec) according to the manufacturer's instructions. The stained cells were then analyzed in a NucleoCounter NC-3000 image cytometer (ChemoMetec).

2.5. Vaccination of Mice Followed by Bacterial Challenge. Sixweek-old female C57BL6/J mice were purchased from DBL (Korea) and housed under specific pathogen-free conditions. At the age of 8 weeks, the mice received an intraperitoneal injection (200 $\mu \mathrm{g}$ in PBS) of S. pneumoniae BAA-255 EMVs. This was repeated twice at intervals of 2 weeks. Two weeks after the third immunization, the mice were infected intraperitoneally with a lethal dose of nonpathogenic $S$. pneumoniae BAA-255 $\left(1 \times 10^{8} \mathrm{cfu}\right)$ or pathogenic S. pneumoniae KCCM-41569 $\left(1 \times 10^{3} \mathrm{cfu}\right)$. Survival was monitored for 7 days. Control mice were immunized with equivalent volumes of PBS and then challenged. All animal experiments were reviewed and approved by the Animal Ethics Committee at the Korea Basic Science Institute (approval number KBSIAEC 1314).

2.6. Identification of the Proteins in EMVs. The proteins in $S$. pneumoniae EMVs were identified by using one-dimensional electrophoresis-liquid chromatography tandem mass spectrometry (1-DE-LC-MS/MS) as described previously [7]. In brief, purified EMVs were lysed and the protein lysates were separated by $12 \%$ sodium dodecyl sulfate-polyacrylamide gel electrophoresis (SDS-PAGE), followed by tryptic in-gel digestion. The digested peptide fractions were then loaded onto a $10 \mathrm{~cm} \times 75 \mu \mathrm{m}$ inner diameter $\mathrm{C} 18$ reverse-phase column (PROXEON, Odense, Denmark; Aqua; particle size, $5 \mu \mathrm{m})$ and subjected to a flow rate of $120 \mathrm{~nL} / \mathrm{min}$. The peptides were eluted with a gradient of $0-80 \%$ acetonitrile containing $0.1 \%$ formic acid for $80 \mathrm{~min}$. All MS and MS/MS spectra were acquired by using a Thermo Finnigan LTQ mass spectrometer (San Jose, CA, USA). Each full MS $(m / z$ range of 400-2,000) scan was followed by three MS/MS scans of the most abundant precursor ions in the MS spectrum, with dynamic exclusion enabled. Proteins were 


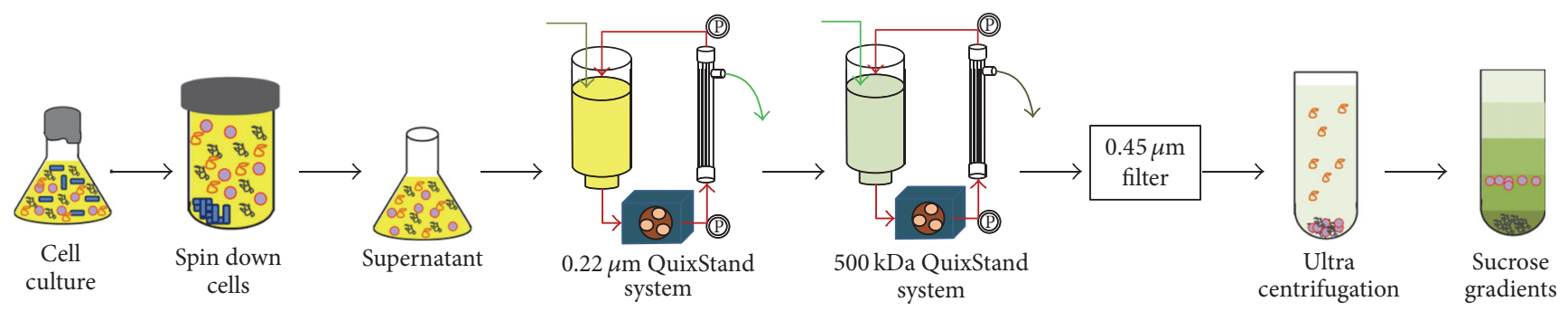

(a)
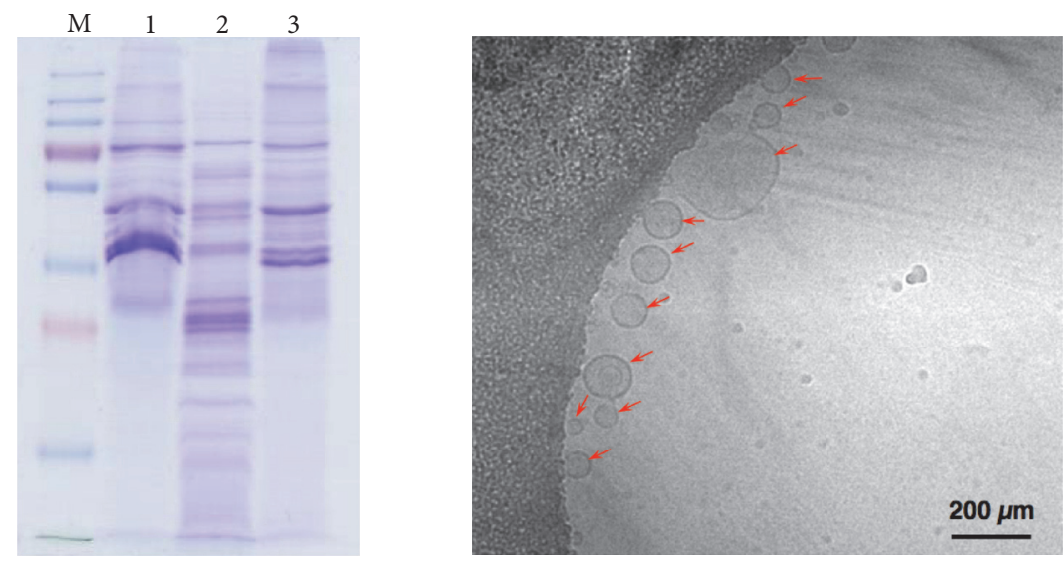

M: protein size marker

1: total lysate before sucrose gradient

2 : sucrose gradient $0.6 \mathrm{M}$ fraction

3 : sucrose gradient $0.6-1.6 \mathrm{M}$ fraction

(b)

(c)

Figure 1: Purification of Streptococcus pneumoniae BAA-255 extracellular membrane vesicles (EMVs). (a) Summary of the method used to prepare S. pneumoniae BAA-255 EMVs. (b) SDS-PAGE of the EMVs before and after sucrose gradient fractionation. (c) Transmission electron microscopy of the EMVs. Red arrows indicate EMVs.

identified by using MASCOT software (ver. 2.4; Matrix Science Inc., USA). A S. pneumoniae R6 protein database (https://www.ncbi.nlm.nih.gov/) was used to analyze the MS/MS data. Carbamidomethylation of cysteine ( $+57 \mathrm{Da})$, oxidation of methionine $(+16 \mathrm{Da})$, and propionamide of cysteine $(+71 \mathrm{Da})$ were considered to be variable protein modifications. The exponentially modified protein abundance index (emPAI) was generated by using MASCOT software and the $\mathrm{mol} \%$ was calculated according to emPAI values. Each sample underwent the MS/MS analysis three times.

\section{Results}

3.1. Production of EMVs from S. pneumoniae. We first examined whether $S$. pneumoniae produced EMVs. Thus, $S$. pneumoniae BAA-255 was grown in Todd-Hewitt broth that was supplemented with $0.5 \%$ yeast extract until the late exponential phase $\left(\mathrm{OD}_{600} \sim 1.0\right)$. The bacterial cells were precipitated by centrifugation and the supernatant was prepared. The supernatant was ultrafiltered to remove cells and cellular debris and sucrose gradient centrifugation was performed to remove contamination with other protein complexes (Figure 1(a)). The EMVs were enriched between 0.6 and 1.6 M sucrose (Figure 1(b)). TEM examination confirmed the presence of S. pneumoniae BAA-255 EMVs. The diameter of the EMVs ranged from $40 \mathrm{~nm}$ to $200 \mathrm{~nm}$ (Figure 1(c)). This is similar to the diameters of other bacterial EMVs.

3.2. Cytotoxic Effects of S. pneumonia EMVs. The endotoxic activity of virulence factors in EMVs significantly hampers their usefulness as vaccines. Therefore, we assessed whether nonpathogenic S. pneumoniae BAA-255 and its EMVs induce host cell damage by treating A549 cells with various concentrations of $S$. pneumoniae BAA-255 bacteria and EMVs. Cell viability and apoptosis assays showed that high concentrations $\left(1 \times 10^{8} \mathrm{cfu}\right.$, MOI 1,000) of $S$. pneumoniae BAA-255 cells slightly reduced cell viability and induced apoptosis (Figures 2(a) and 2(b)). However, when the cells were treated with EMVs from $S$. pneumoniae BAA255 , cell viability and apoptosis rates were unchanged, even in the presence of high concentrations $(200 \mu \mathrm{g})$ of EMVs (Figures 2(c) and 2(d)). By contrast, a pathogenic strain of S. pneumoniae, namely, KCCM-41569, killed A549 cells even at very low concentrations $\left(1 \times 10^{3} \mathrm{cfu}\right.$, MOI 0.01) (Figure S1) (see Supplementary Material available online at https://doi.org/10.1155/2017/7931982). These results suggest that EMVs from nonpathogenic S. pneumoniae BAA-255 are not cytotoxic. 


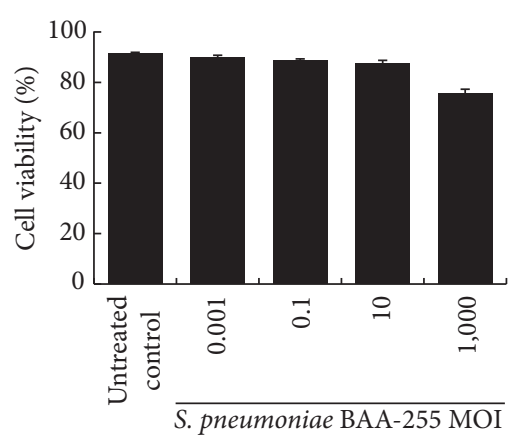

(a)

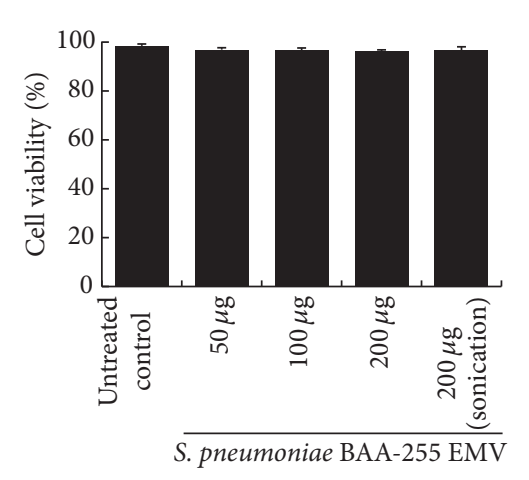

(c)
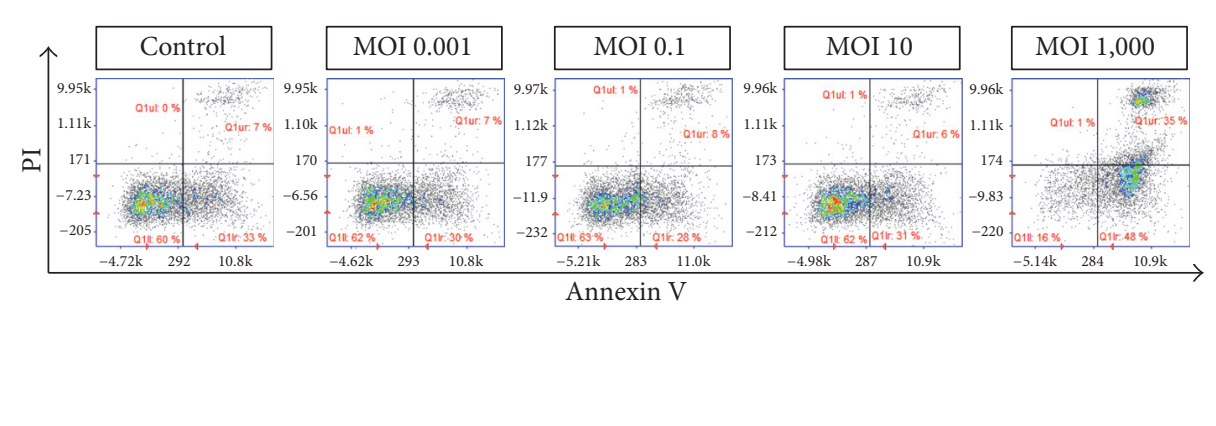

Annexin V

(b)
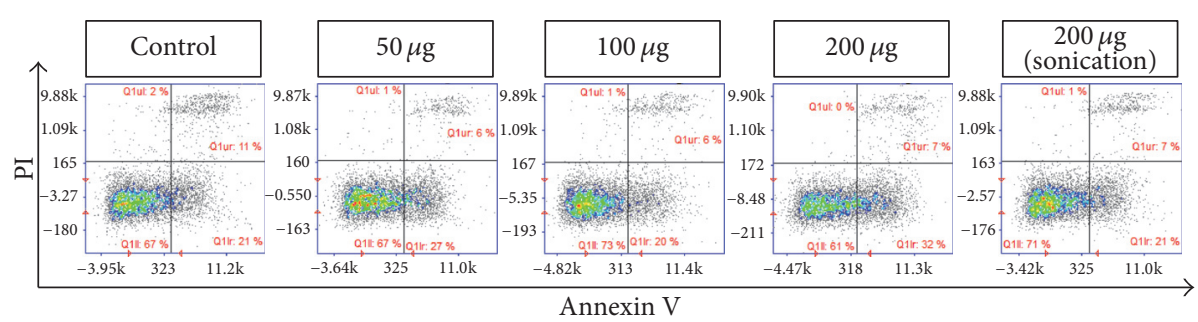

(d)

FIgure 2: Cytotoxicity of intact $S$. pneumoniae BAA-255 and its EMVs. A549 cells were treated with various concentrations of intact $S$. pneumoniae BAA-255 and cell viability (a) and apoptosis (b) were analyzed. A549 cells treated with various concentrations of S. pneumoniae BAA-255 EMVs and cell viability (c) and apoptosis (d) were analyzed.

3.3. Immunization with S. pneumoniae EMVs. To evaluate the potential and efficacy of EMVs as a new vaccine against $S$. pneumoniae infection, mice were vaccinated with $S$. pneumoniae BAA-255 EMVs and then challenged with live S. pneumoniae BAA-255 bacteria. After bacterial challenge, $10 \%$ of the control mice survived. By contrast, $60 \%$ of the EMV-vaccinated and challenged mice survived (Figure 3(a)). This suggests that vaccination with $S$. pneumoniae EMVs protects mice against $S$. pneumoniae. Control and EMVvaccinated mice were also challenged with KCCM-41569, a pathogenic strain of S. pneumoniae. None of the control mice survived infection with $1 \times 10^{3} \mathrm{cfu}$ of $S$. pneumoniae KCCM41569. However, some of the vaccinated mice did survive: $40 \%$ survived after three immunizations and 20\% survived after a single or double immunization (Figure 3(b)). These results show that immunization with EMVs isolated from a nonpathogenic $S$. pneumoniae BAA-255 strain not only protected against homologous challenge but also provided crossprotection against challenge with a pathogenic heterologous strain.

3.4. Identification of S. pneumoniae EMV Proteins. LC-based proteomic analysis was performed to identify the protein components of S. pneumoniae EMVs. 1D-LC-MS/MS analysis identified a total of 104 proteins in S. pneumoniae BAA-255 EMVs (Supplementary Table S1). A cell location prediction program was then used to determine the subcellular localization of the identified proteins. Of the 104 proteins identified in S. pneumoniae EMVs, 32 were extracellular, 28 were from the membrane, one was from the cell wall, and 43 were cytoplasmic (Figure 4(a)). Of the 104 identified proteins, the 32 extracellular proteins accounted for more than half $(67.2 \%)$ of the total protein in S. pneumoniae EMVs (Figure 4(b)).

The identified proteins were then analyzed by using a bioinformatics tool (Clusters of Orthologous Groups (COGs)) to determine the putative function of S. pneumoniae EMVs. The proteins in S. pneumoniae EMVs were mainly involved in the transport and metabolism of biomaterials such as amino acids and carbohydrates, as well as inorganic ions (Figure 5).

\section{Discussion}

Vaccines that prevent viral and bacterial diseases significantly improve public health. There are three types of vaccines against these microorganisms, namely, attenuated live vaccines, inactivated vaccines, and subunit vaccines. Although attenuated live and inactivated vaccines provide high levels of protection against viral and bacterial disease, there are concerns that they also elicit toxic side effects due to the presence of virulence factors. While this problem is overcome by subunit vaccines, they in turn are less effective and more expensive to produce than attenuated live or inactivated vaccines [8]. One way to sidestep these problems with existing antibacterial vaccine approaches is to use bacterial EMVs, as these are safe, cheap to produce, and provide 


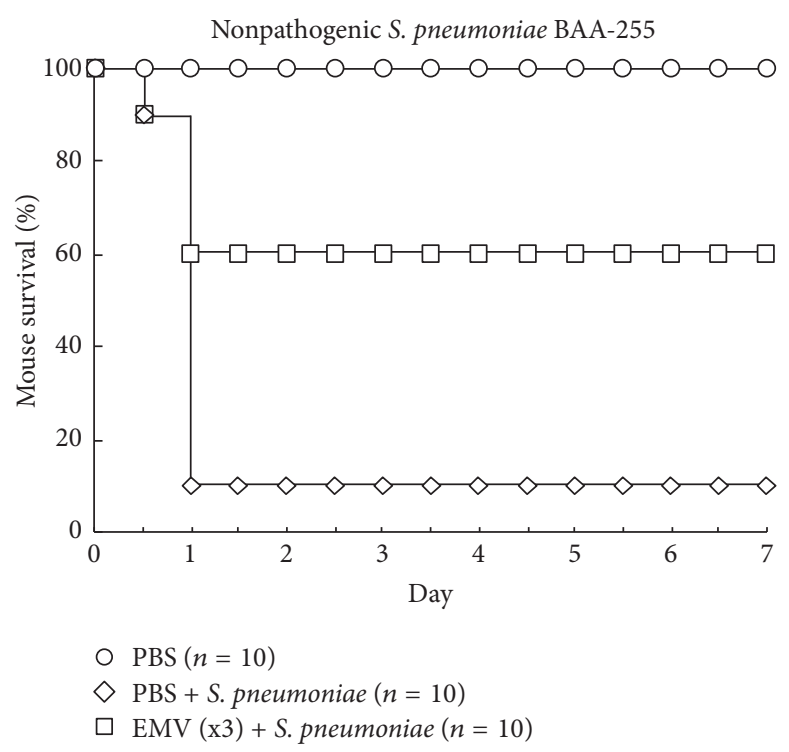

(a)

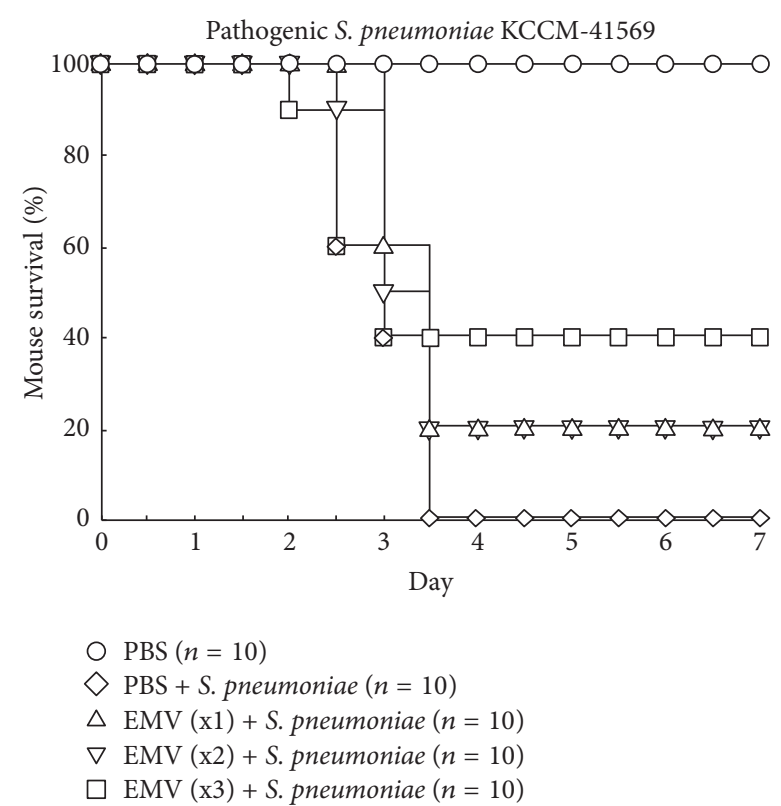

(b)

FIGURE 3: Survival of vaccinated mice after infection with Streptococcus pneumoniae. Mice (8 weeks old) were vaccinated intraperitoneally with S. pneumoniae BAA-255 extracellular membrane vesicles. After intraperitoneal inoculation with $1 \times 10^{8}$ cfu of nonpathogenic $S$. pneumoniae BAA-255 or $1 \times 10^{3}$ cfu of pathogenic S. pneumoniae KCCM-41569, survival over 7 days was assessed. Equivalent volumes of phosphatebuffered saline served as a vaccine control.

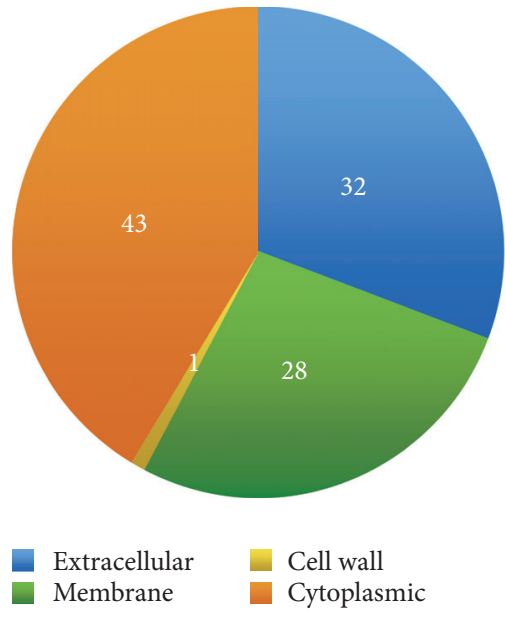

(a)

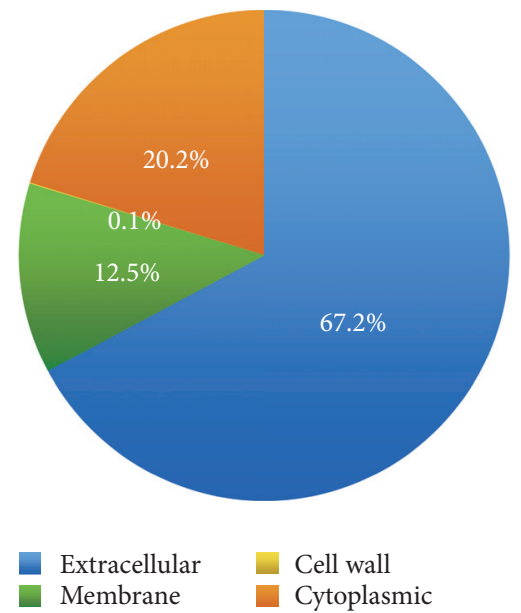

(b)

FIGURE 4: Subcellular localization of proteins identified in Streptococcus pneumoniae BAA-255 extracellular membrane vesicles (EMVs). The subcellular localization of the different proteins identified in the EMVs according to the number of proteins (a) or the total amount of protein (b) is shown. The subcellular localization of the proteins was determined by using CELLO (http://cello.life.nctu.edu.tw).

high levels of protection [9]. Indeed, several EMV vaccines against serogroup B Neisseria meningitides (MenB) have been licensed for human use in Norway, Cuba, Chile, and New Zealand [10-13]. Here, we showed that S. pneumoniae EMVs are both effective against $S$. pneumoniae infection and safe in an animal model (Figures 2 and 3). This supports the notion that bacterial EMVs have great potential as next-generation vaccines that protect humans from bacterial infections.
We identified 61 putative antigenic proteins (extracellular, membrane, and cell wall proteins) that may be recognized by the host immune system and induce adaptive immunity. Previous reports identified the immunogenic proteins in exoproteomes of $S$. pneumoniae $[14,15]$. We also found some of these immunogenic proteins in S. pneumoniae EMVs, namely, MalX, AliA, Ami, PspA, Eno, ABC-SBP, Sphra, and ZamB (Supplementary Table S1). These results suggest that 

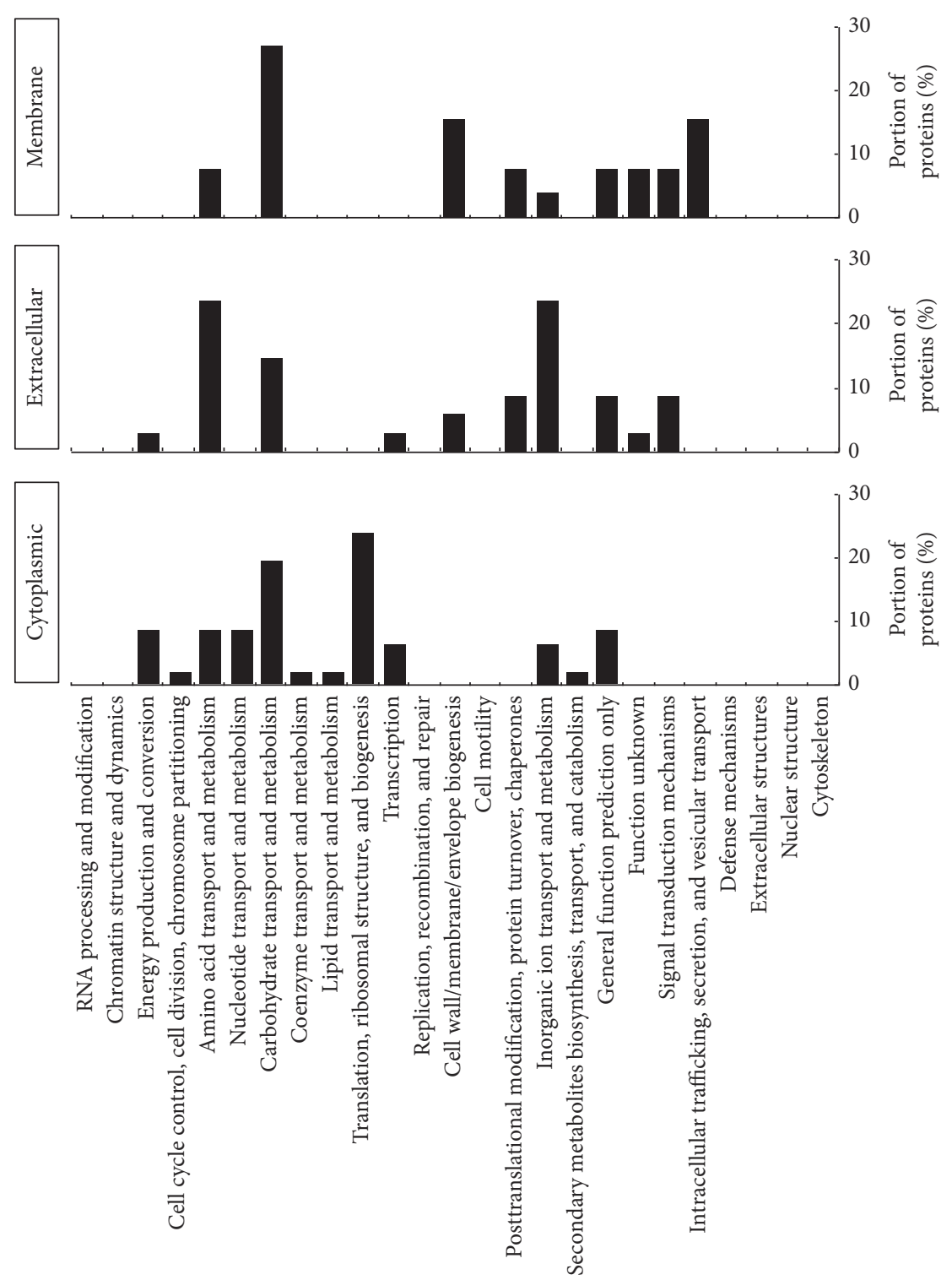

FIGURE 5: Functional annotation of the Streptococcus pneumoniae extracellular membrane vesicle proteins. The proteins were clustered according to their putative functions, which were determined by COGs (Clusters of Orthologous Groups).

the proteins in the EMVs may be immunogenic antigens that induce the production of specific antibodies by the host. Previously, existence of EMVs from other Gram-positive bacteria and their protein composition was reported. 90 proteins from Staphylococcus aureus EMVs [16], 104 proteins from Bacillus anthracis EMVs [17], and 426 proteins were identified from Clostridium perfringens EMVs [18]. Among the immunogenic proteins identified in S. pneumoniae, only MalX, Eno, and Sphra were discovered in C. perfringens. Therefore, these results suggest that the other immunogenic proteins may be specific for S. pneumonia and each bacterium has different immunogenic protein sets. These proteins can be used to develop diagnostic kits and subunit vaccines for $S$. pneumoniae.

In this study, we isolated the EMVs from a nonpathogenic S. pneumoniae strain (BAA-255), which has no polysaccharide capsule. The main difference between pathogenic and nonpathogenic S. pneumoniae is the presence of a polysaccharide capsule in the former. This polysaccharide capsule is the most potent virulence factor [19]. Capsular polysaccharide is also used as pneumococcal vaccines. Our results showed that immunization with the EMVs from the nonpathogenic $S$. pneumoniae strain BAA-255 protected mice from infection with a pathogenic $S$. pneumoniae strain (KCCM-41569), especially when multiple vaccinations were given (Figure 3(b)). This suggests that EMV proteins are responsible for acquired immunity against bacterial infection. For immunization, we inoculated EMVs only. Therefore, less EMVs would be sufficient for immunization when they are inoculated with adjuvants.

S. pneumoniae BAA-255 is a nonpathogenic noncapsulated strain and we found that its EMVs had no cytotoxic effect on A549 lung cancer cells, even at high concentrations $(200 \mu \mathrm{g})$ (Figure 2). In fact, the EMVs from nonpathogenic 
S. pneumoniae BAA-255 was even safer than the EMVs from the environmental soil bacterium Pseudomonas putida KT2440: a previous study showed that $25 \mu \mathrm{g}$ of $P$. putida KT2440 EMVs induced early apoptosis in A549 cells [7]. The difference between $S$. pneumoniae and $P$. putida EMVs with respect to cytotoxicity may be due to the fact that $P$. putida is Gram-negative: the EMVs from Gram-negative bacteria often express endotoxic lipopolysaccharide (LPS) on their outer membrane (these EMVs are commonly known as outer membrane vesicles) [20]. Indeed, EMVs from many Gramnegative bacteria are cytotoxic [21]. Since S. pneumoniae BAA-255 is Gram-positive, its EMVs do not carry LPS. Thus, the EMVs of Gram-positive bacteria may be safer and more suitable for vaccines than EMVs derived from Gram-negative bacteria.

\section{Conclusions}

The present study aimed to examine the potential of bacterial EMVs as vaccines. The results showed that EMVs isolated from S. pneumoniae BAA-255 protected mice with no notable side effects. In addition, we identified the immunogenic proteins that are expressed on S. pneumoniae BAA-255 EMVs. Since EMVs are more immunogenic than an equivalent amount of bacterial cell extract [15], these findings suggest that EMVs are highly promising as potential vaccine antigens.

\section{Competing Interests}

The authors declare that they have no competing interests.

\section{Authors' Contributions}

Chi-Won Choi and Edmond Changkyun Park contributed equally to this work.

\section{Acknowledgments}

This research was supported by the Korea Basic Science Institute Research Program (D36402), the Science Research Center Program (2015R1A5A1009024), and the Basic Science Research Program (2015R1C1A1A01054897) of the National Research Foundation (NRF) funded by the Ministry of Science, ICT and Future Planning, and the Korea Health Technology R\&D Project (HI14C2726, HI16C0950) through the Korea Health Industry Development Institute (KHIDI) funded by the Ministry of Health \& Welfare.

\section{References}

[1] R. A. Hirst, H. Yesilkaya, E. Clitheroe et al., "Sensitivities of human monocytes and epithelial cells to pneumolysin are different," Infection and Immunity, vol. 70, no. 2, pp. 1017-1022, 2002.

[2] S. J. Kelly, K. B. Taylor, S. Li, and M. J. Jedrzejas, "Kinetic properties of Streptococcus pneumoniae hyaluronate lyase," Glycobiology, vol. 11, no. 4, pp. 297-304, 2001.
[3] T. J. Mitchell, "Virulence factors and the pathogenesis of disease caused by Streptococcus pneumoniae," Research in Microbiology, vol. 151, no. 6, pp. 413-419, 2000.

[4] M. van der Linden, A. Al-Lahham, W. Nicklas, and R. R. Reinert, "Molecular characterization of pneumococcal isolates from pets and laboratory animals," PLOS ONE, vol. 4, no. 12, Article ID e8286, 2009.

[5] G. Oligbu, Y. Hsia, L. Folgori, S. Collins, and S. Ladhani, "Pneumococcal conjugate vaccine failure in children: a systematic review of the literature," Vaccine, vol. 34, no. 50, pp. 6126-6132, 2016.

[6] A. Kulp and M. J. Kuehn, "Biological functions and biogenesis of secreted bacterial outer membrane vesicles," Annual Review of Microbiology, vol. 64, pp. 163-184, 2010.

[7] C.-W. Choi, E. C. Park, S. H. Yun et al., "Proteomic characterization of the outer membrane vesicle of Pseudomonas putida KT2440," Journal of Proteome Research, vol. 13, no. 10, pp. 4298 4309, 2014.

[8] E. N. T. Meeusen, J. Walker, A. Peters, P.-P. Pastoret, and G. Jungersen, "Current status of veterinary vaccines," Clinical Microbiology Reviews, vol. 20, no. 3, pp. 489-510, 2007.

[9] G.-H. Kim, C. W. Choi, E. C. Park, S.-Y. Lee, and S. I. Kim, "Isolation and proteomic characterization of bacterial extracellular membrane vesicles," Current Protein and Peptide Science, vol. 15, no. 7, pp. 719-731, 2014.

[10] G. Bjune, E. A. Hoiby, J. K. Gronnesby et al., "Effect of outer membrane vesicle vaccine against group B meningococcal disease in Norway," The Lancet, vol. 338, no. 8775, pp. 1093-1096, 1991.

[11] D. R. Martin, S. J. Walker, M. G. Baker, and D. R. Lennon, "New Zealand epidemic of meningococcal disease identified by a strain with phenotype B:4:P1.4," Journal of Infectious Diseases, vol. 177, no. 2, pp. 497-500, 1998.

[12] G. V. Sierra, H. C. Campa, N. M. Varcacel et al., "Vaccine against group B Neisseria meningitidis: protection trial and mass vaccination results in Cuba," NIPH Annals, vol. 14, no. 2, pp. 195-210, 1991.

[13] J. Boslego, J. Garcia, C. Cruz et al., "Efficacy, safety, and immunogenicity of a meningococcal group B (15:P1.3) outer membrane protein vaccine in Iquique, Chile," Vaccine, vol. 13, no. 9, pp. 821-829, 1995.

[14] C.-W. Choi, Y. G. Lee, S.-O. Kwon et al., "Analysis of Streptococcus pneumoniae secreted antigens by immuno-proteomic approach," Diagnostic Microbiology and Infectious Disease, vol. 72, no. 4, pp. 318-327, 2012.

[15] A. Olaya-Abril, R. Prados-Rosales, M. J. McConnell et al., "Characterization of protective extracellular membranederived vesicles produced by Streptococcus pneumoniae," Journal of Proteomics, vol. 106, pp. 46-60, 2014.

[16] E.-Y. Lee, D.-Y. Choi, D.-K. Kim et al., "Gram-positive bacteria produce membrane vesicles: proteomics-based characterization of Staphylococcus aureus-derived membrane vesicles," Proteomics, vol. 9, no. 24, pp. 5425-5436, 2009.

[17] J. Rivera, R. J. B. Cordero, A. S. Nakouzi, S. Frases, A. Nicola, and A. Casadevall, "Bacillus anthracis produces membrane-derived vesicles containing biologically active toxins," Proceedings of the National Academy of Sciences of the United States of America, vol. 107, no. 44, pp. 19002-19007, 2010.

[18] Y. Jiang, Q. Kong, K. L. Roland, and R. Curtiss, "Membrane vesicles of Clostridium perfringens type A strains induce innate and adaptive immunity," International Journal of Medical Microbiology, vol. 304, no. 3-4, pp. 431-443, 2014. 
[19] A. M. Mitchell and T. J. Mitchell, "Streptococcus pneumoniae: virulence factors and variation," Clinical Microbiology and Infection, vol. 16, no. 5, pp. 411-418, 2010.

[20] B. van de Waterbeemd, M. Streefland, P. van der Ley et al., "Improved OMV vaccine against Neisseria meningitidis using genetically engineered strains and a detergent-free purification process," Vaccine, vol. 28, no. 30, pp. 4810-4816, 2010.

[21] T. N. Ellis and M. J. Kuehn, "Virulence and immunomodulatory roles of bacterial outer membrane vesicles," Microbiology and Molecular Biology Reviews, vol. 74, no. 1, pp. 81-94, 2010. 


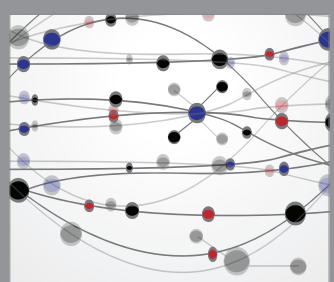

The Scientific World Journal
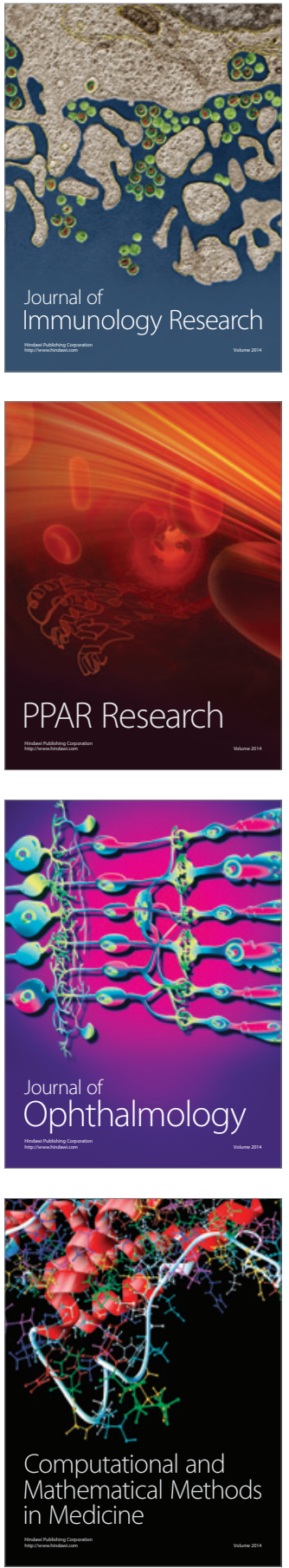

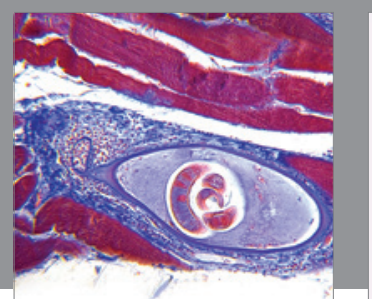

Gastroenterology Research and Practice
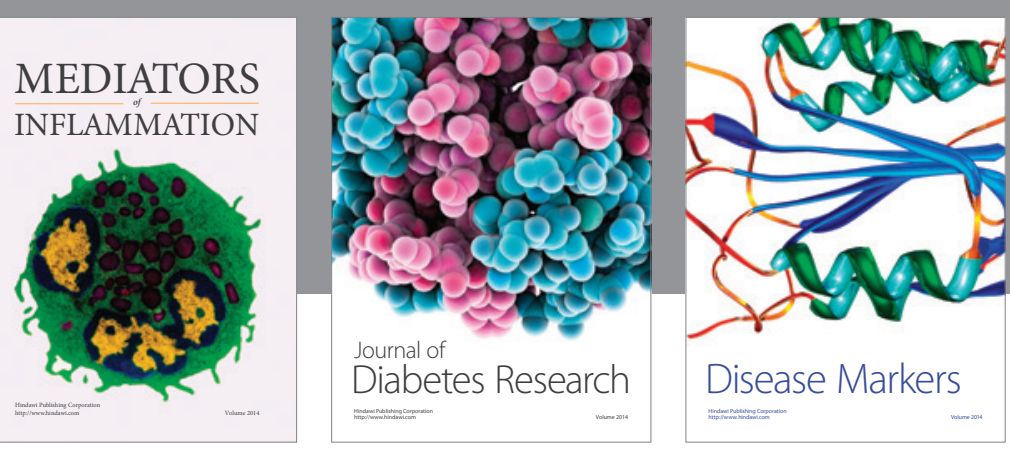

Disease Markers

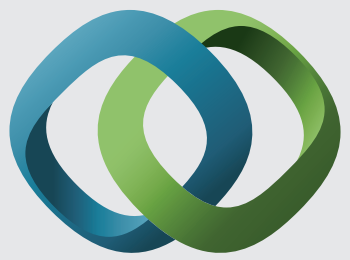

\section{Hindawi}

Submit your manuscripts at

https://www.hindawi.com
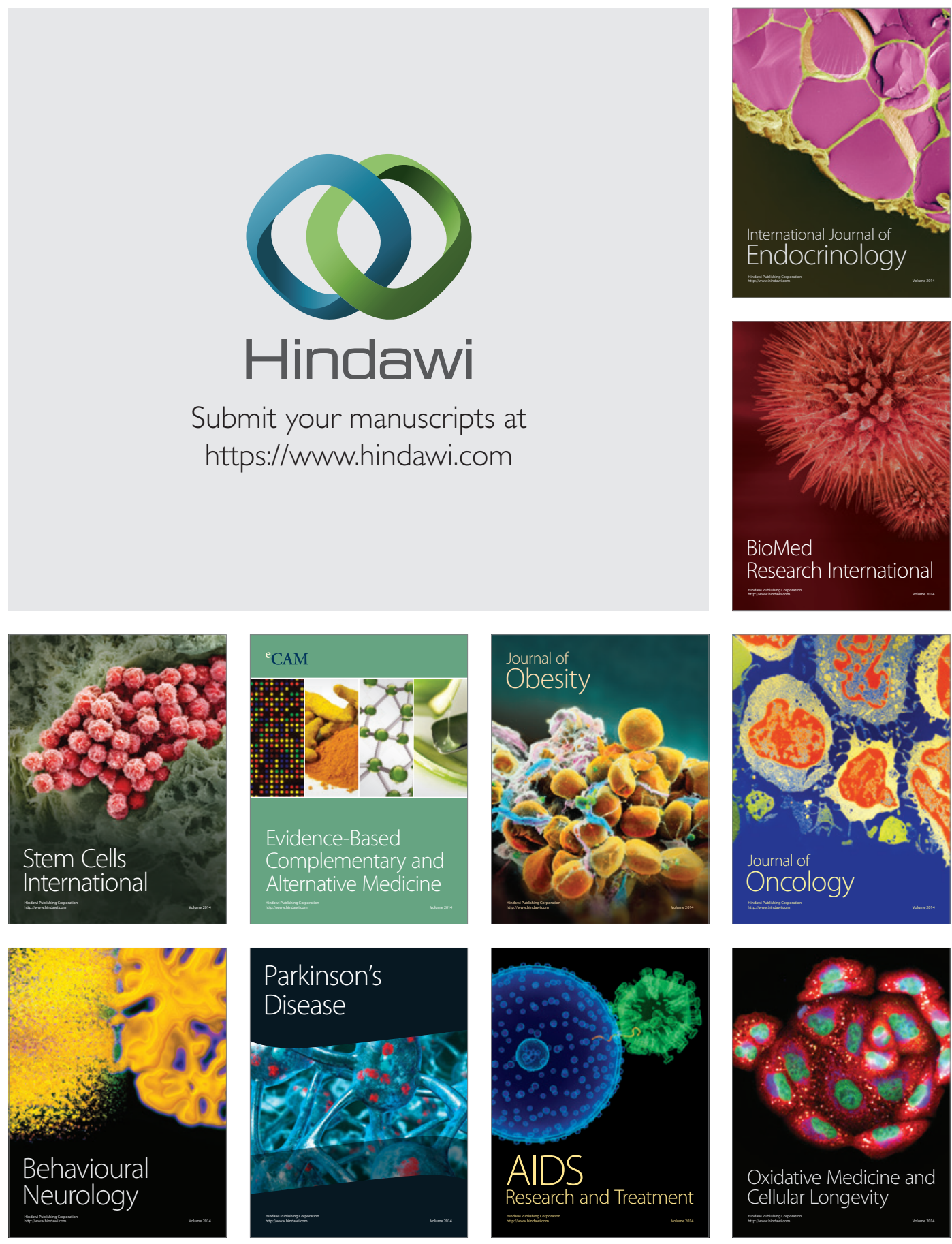